\title{
Research on reliability evaluation of agricultural expert experience knowledge
}

\author{
Dai xiaopeng ${ }^{\mathrm{a}} \mathrm{Li}_{\text {donghui }}{ }^{\mathrm{b}}$, \\ School of information Science \& Technology of Hunan Agricultural University ChangSha China \\ adaixpedu@126.com, bdoner@263.net
}

Keywords: Agricultural experts; Empirical knowledge; Evidence theory; Reliability evaluation Abstract: Agricultural expert experience highlights its advantages in the agricultural biological disaster forecast evaluation. Aiming at solving the expert experience fusion, this paper converted expert information into reliability probability density distribution, determined the evidence theory knowledge framework and basic probability distribution function based on the consistency definition and at the same time, the weight of each distribution is confirmed by using D-S synthesis rules. This paper ultimately obtained after-experience reliability level of fusion expert experiences in the agricultural production.

\section{Introduction}

In the agricultural biological disaster forecast evaluation, objective test data is difficult to be obtained for the factors limitation such as funds and time. While the traditional reliability evaluation method uses mathematical statistics large sample theory as its foundation, this method can't be suitable for agricultural biological disasters reliability evaluation. And plant protection experts, farmers and related domain experts accumulated a large amount of knowledge during their everyday study. Their experts experiences can better reflect the actual situation of agricultural biological disaster so that expert experiences should be emphasized heavily. In order to get higher credibility results needs carry on the expert experience fusion according to many experts' judgments ${ }^{[1,2]}$

Evidence theory is a new important kind of developed uncertainty reasoning method, suitable for decisions on human existence and uncertain factors. It has obvious advantages in the treatment of the subjective judgment and the uncertain knowledge synthesis ${ }^{[3]}$. Booker discussed expert information extraction and the application of fuzzy theory ${ }^{[4,5]}$; Dey described the method of transforming experts' information with quantile formal description to the probability distribution ${ }^{[6]}$; Szwed, Hora and Bram researched on different forms of expert information fusion. The evidence theory should be widely applied on reliability evaluation of the agricultural biological disaster prediction.

\section{Evidence theory-based expert information fusion}

\section{D-S synthetic rules}

Set BEL1 and BEL2 as two trust function on the same recognition framework $\Theta, \mathrm{m} 1, \mathrm{~m} 2$ is the corresponding basic probability assignment, focal elements are $\mathrm{A} 1, \mathrm{~A} 2, \ldots \mathrm{Ak}$, and $\mathrm{B} 1, \mathrm{~B} 2, \ldots \mathrm{Br}$, then $\mathrm{D}$ - $\mathrm{S}$ synthetic rules for two evidence is ${ }^{[11,12]}$.

$$
\begin{aligned}
& m(\boldsymbol{C})= \begin{cases}\frac{\sum_{\boldsymbol{A}_{i} \cap \boldsymbol{B}_{j} \neq \boldsymbol{\Phi}} m_{1}\left(\boldsymbol{A}_{i}\right) m_{2}\left(\boldsymbol{B}_{j}\right)}{1-K_{1}} & \forall \boldsymbol{C} \subset \boldsymbol{U}, \boldsymbol{C} \neq \boldsymbol{\Phi} \\
0 & \boldsymbol{C} \neq \boldsymbol{\Phi}\end{cases} \\
& K_{1}=\sum_{\substack{i, j \\
\boldsymbol{A}_{i} \cap \boldsymbol{B}_{j}=\boldsymbol{\Phi}}} m_{1}\left(\boldsymbol{A}_{i}\right) m_{2}\left(\boldsymbol{B}_{j}\right)<1
\end{aligned}
$$




\section{The transformation of the expert experience to the probability distribution}

In the agricultural biological disaster evaluation, the relevant field experts gave an estimate value of agricultural biological disasters rely on their experience. The evaluation is based on the weather conditions, the ecological environment, historical data and crop cultivation mode. Assuming each expert can be trusted, so all the reliability estimates are around the true value. And the more close to the true value, the more likelihood of the valuation fall in this interval. Suppose it obey the normal distribution $\mathrm{N}(\mu, \delta 2)$. Scene, experts often given reliability of interval estimation: the reliability interval estimation of base evaluation is ( $R L$, RU ), when the confidence is $1-\alpha$, i.e., $P(R L<R<R U)=1-\alpha$, as shown in figure 1.

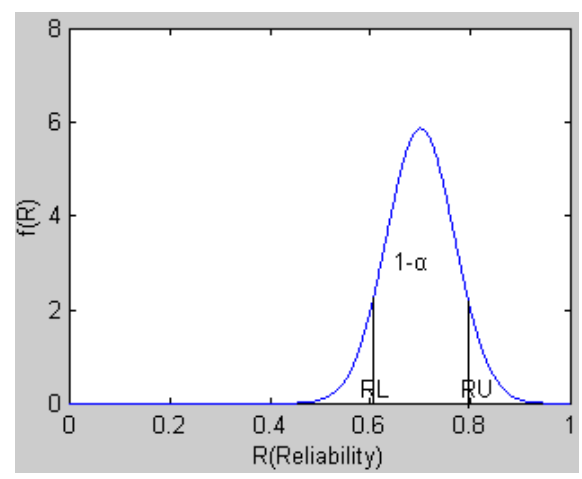

Fig.1 Transformation probability density distribution

So the experts-given interval estimation equals to the reliability obeys distribution $\mathrm{N}(\mu, \delta 2)$, PDF for $f(R)$, and the possibility of $R$ falls among the interval (RL, RU) is 1- $\alpha$, namely for surrounded area between probability density curve and the interval (RL, RU). Through this method, it converted the expert-experience interval estimation into probability density distribution.

The distribution has two unknown parameter-- $\mu, \delta 2$. Normal distribution is symmetrical, and the value of mean $\mu$ is the neutral value of valuation interval

$$
\mu=\frac{R_{L}+R_{U}}{2}
$$

Variance $\delta 2$ can be calculated according to the interval estimation

$$
\int_{R_{L}}^{R_{U}} f(R) d R=1-\alpha
$$

\section{Information consistency}

To the above description, the $\mathrm{i}, \mathrm{j}$ experts gives two kinds of interval estimation, which obey distribution $\mathrm{N}(\mu \mathrm{i}, \delta 2 \mathrm{i}), \mathrm{N}(\mu \mathrm{j}, \delta 2 \mathrm{j})$, and the probability density function is respectively fi ( $\mathrm{R}), \mathrm{fj}(\mathrm{R}$ ), as shown in figure 2. Two distributions have coincidence parts.

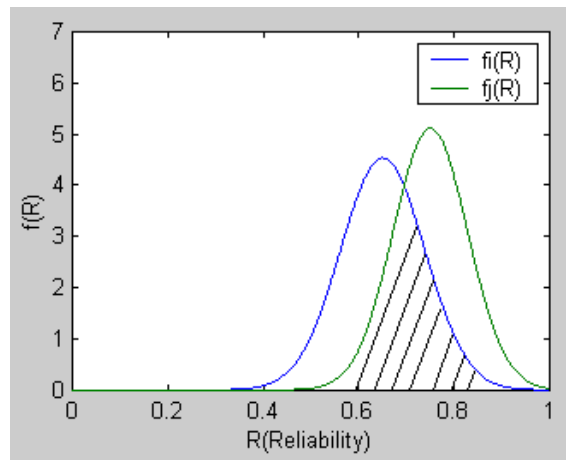

Fig.2 Experts consistency 
Definition: Converting two experts-given reliability interval estimation into probability distribution for $\mathrm{fi}(\mathrm{R}), \mathrm{fj}(\mathrm{R})$, so the expert information consistency is the ratio of the intersection and the set for two probability distribution and transverse surrounded area, namely

$$
C M\left(f_{i}(R), f_{j}(R)\right)=\frac{\int_{0}^{1} \min \left(f_{i}(R), f_{j}(R)\right) d R}{\int_{0}^{1} \max \left(f_{i}(R), f_{j}(R)\right) d R}
$$

$C M\left(f_{i}(R), f_{j}(R)\right)$ also as $C M_{i j}$ can express the support degree of one expert to another one. The higher the value, the higher consensus degree of the two experts and so the higher credibility of the two experts' information's consistent part.

From the definition, it is easy to see the consistency with the nature

$$
C M_{i j}=C M_{j i}
$$

\section{Information fusion based on the D-S theory}

$\mathrm{N}$ experts evaluated agricultural biological disasters, each expert evaluation results for $\mathrm{Ei}(\mathrm{I}=1$, $2, \ldots, \mathrm{n})$, Ei can be converted to probability density distribution fi (R). From the consistency definition, any two expert information $\mathrm{Ei}, \mathrm{Ej}$ have a consistent degree, and then we can combine each two of all the expert information and get the $\mathrm{n} \times \mathrm{n}$ consistency matrix

$$
\boldsymbol{C M}=\left[\begin{array}{cccc}
1 & C M_{12} & \ldots & C M_{1 n} \\
C M_{21} & 1 & \ldots & C M_{2 n} \\
\cdots & & \ldots & \ldots \\
C M_{n 1} & C M_{n 2} & \ldots & 1
\end{array}\right]
$$

According to the consistency nature, the consistency matrix is symmetric matrix, and all the elements on the principal diagonal are 1.

Each element in the line of the matrix is normalized, and got a new matrix $\boldsymbol{C M}$

$$
C M_{i j}^{\prime}=\frac{C M_{i j}}{\sum_{j=1}^{n} C M_{i j}} \quad(i, j=1,2, \ldots, n)
$$

All the after-transformation experts information formed the probability density distribution set $\{\mathrm{Sj}(\mathrm{R}) \mid \mathrm{j}=1,2 \ldots, \mathrm{n}\}$, and as an identified frame $\Theta, C M_{i j}^{\prime}$ expresses the probability that the i expert think R obey the probability density distribution $\mathrm{fj}(\mathrm{R})$ is true. Structure basic probability density distribution function $\mathrm{m}: 2 \Theta->[0,1]$

$$
\begin{cases}m_{i}(A)=C M_{i j}^{\prime} & A=f_{j}(R) \\ m_{i}(A)=0 & A \neq f_{j}(R), A \subseteq \boldsymbol{\Theta}\end{cases}
$$

Using the evidence theory synthesis rules, after $n-1$ synthesis

$$
m(A)= \begin{cases}\frac{\prod_{i=1}^{n} C M_{i j}^{\prime}}{1-\sum_{j=1}^{n} \prod_{i=1, i \neq j}^{n} C M_{i j}^{\prime}} & A=f_{j}(R) \quad i, j=1,2 \ldots n \\ 0 & A \neq f_{j}(R), A \subseteq \Theta\end{cases}
$$

$w_{j}=m\left(f_{j}(R)\right) \quad(\mathrm{j}=1,2, \ldots, \mathrm{n})$ represents the after-fusion probability density distribution weight. 


\section{Problems reliability}

The probability density distribution is the interval estimation transformation of the expert experience reliability, namely the weight of probability density distribution is the weight of expert experience. So the system reliability is

After-fusion reliability distribution is

$$
R_{s}=w_{1} \cdot \mu_{1}+w_{2} \cdot \mu_{2}+\ldots+w_{n} \cdot \mu_{n}
$$

$$
f_{s}=w_{1} \cdot f_{1}+w_{2} \cdot f_{2}+\ldots+w_{n} \cdot f_{n}
$$

\section{Expert information discount}

Different experts have different credibility on agricultural biological disasters. If it has a certain understanding of its trust degree, then discount it before the fusion of each expert's information.

Assuming the credibility of the I expert is $\beta \mathrm{i}$, the basic probability distribution function of the expert support proposition A is mi ( A ), then the after-discount basic probability distribution function $m$ is

$$
m_{i}^{\prime}(A)=\beta_{i} \cdot m_{i}(A)
$$

According to the basic probability distribution function, for above D-S rules synthesis, get the probability distribution weight.

1. Instance analysis

Assuming in an agricultural biological disaster evaluation, four experts gave prediction reliability interval estimation, $\quad \mathrm{P} 1(0.6<\mathrm{R}<0.8)=0.85, \quad \mathrm{P} 2(0.7<\mathrm{R}<0.85)=0.9, \quad \mathrm{P} 3(0.65<\mathrm{R}<0.8)=0.8$, $\mathrm{P} 4(0.7<\mathrm{R}<0.8)=0.8$.

Converted it into probability density distribution, which respectively obeyed normal distribution, the parameters are

$(0.7000,0.06942),(0.7750,0.04572),(0.7250,0.05862),(0.7500,0.03912)$

The consistency matrix as follows

$$
\boldsymbol{C M}_{4 \times 4}=\left[\begin{array}{cccc}
1 & 0.7291 & 0.8579 & 0.5092 \\
0.7291 & 1 & 0.6170 & 0.7596 \\
0.8579 & 0.6170 & 1 & 0.7522 \\
0.5092 & 0.7596 & 0.7522 & 1
\end{array}\right]
$$

The after-normalized new consistency matrix

$$
\boldsymbol{C M}_{4 \times 4}^{\prime}=\left[\begin{array}{llll}
0.3230 & 0.2355 & 0.2771 & 0.1645 \\
0.2348 & 0.3220 & 0.1987 & 0.2446 \\
0.2658 & 0.1912 & 0.3099 & 0.2331 \\
0.1686 & 0.2514 & 0.2490 & 0.3310
\end{array}\right]
$$

First this paper combined the first and the second expert information according to the D-S combination rules. As shown in Table.1.

Table.1 Two experts' information fusion

\begin{tabular}{c|c|c|c|c|c}
\hline \multicolumn{2}{c|}{} & \multicolumn{4}{c}{$\mathrm{m}_{1}(\bullet)$} \\
\cline { 3 - 6 } & $\mathrm{m}_{1}\left(\mathrm{f}_{1}\right)$ & $\mathrm{m}_{1}\left(\mathrm{f}_{2}\right)$ & $\mathrm{m}_{1}\left(\mathrm{f}_{3}\right)$ & $\mathrm{m}_{1}\left(\mathrm{f}_{4}\right)$ \\
\hline \multirow{4}{*}{$\mathrm{m}_{2}(\bullet)$} & $\mathrm{m}_{2}\left(\mathrm{f}_{1}\right)$ & $(0.07584)_{\mathrm{f} 1}$ & $(0.05530)_{\Phi}$ & $(0.06506)_{\Phi}$ & $(0.03862)_{\Phi}$ \\
\cline { 2 - 6 } & $\mathrm{m}_{2}\left(\mathrm{f}_{2}\right)$ & $(0.10401)_{\Phi}$ & $(0.07583)_{\mathrm{f} 2}$ & $(0.08923)_{\Phi}$ & $(0.05297)_{\Phi}$ \\
\cline { 2 - 6 } & $\mathrm{m}_{2}\left(\mathrm{f}_{3}\right)$ & $(0.06418)_{\Phi}$ & $(0.04679)_{\Phi}$ & $(0.05506)_{\mathrm{f} 3}$ & $(0.03269)_{\Phi}$ \\
\cline { 2 - 6 } & $\mathrm{m}_{2}\left(\mathrm{f}_{4}\right)$ & $(0.07901)_{\Phi}$ & $(0.05760)_{\Phi}$ & $(0.06778)_{\Phi}$ & $(0.04024)_{\mathrm{f} 4}$ \\
\hline
\end{tabular}

Factor $\mathrm{K} 1$ is 
$\mathrm{K}_{1}=0.10401+0.06418+0.07901+0.05530+0.04679+0.05760+0.06506+0.08923+0.06778+0.03862+$ $0.05297+0.03269$

This paper got the basic probability assignments based on the two experts' information after fusion.

$$
\begin{aligned}
& m_{12}\left(f_{1}\right)=\frac{0.07584}{1-\mathrm{K}_{1}} \approx 0.3073 \quad m_{12}\left(f_{2}\right)=\frac{0.07583}{1-\mathrm{K}_{1}} \approx 0.3073 \\
& m_{12}\left(f_{3}\right)=\frac{0.05506}{1-\mathrm{K}_{1}} \approx 0.2231 m_{12}\left(f_{4}\right)=\frac{0.04024}{1-\mathrm{K}_{1}} \approx 0.1631
\end{aligned}
$$

After n-1 combination, fusion results as shown in Table.2.

Table.2 D-S fusion results

\begin{tabular}{c|c|c|c|c}
\hline & $\mathrm{f}_{1}$ & $\mathrm{f}_{2}$ & $\mathrm{f}_{3}$ & $\mathrm{f}_{4}$ \\
\hline $\mathrm{m}_{1 \oplus 2}$ & 0.3073 & 0.3073 & 0.2231 & 0.1631 \\
\hline $\mathrm{m}_{1 \oplus 2 \oplus 3}$ & 0.3310 & 0.2381 & 0.2801 & 0.1541 \\
\hline $\mathrm{m}_{1 \oplus 2 \oplus 3 \oplus 4}$ & 0.2384 & 0.2568 & 0.2922 & 0.2188 \\
\hline
\end{tabular}

After the d-s combination, got each distribution weight

$$
\mathrm{w}_{1}=0.2384, \quad \mathrm{w}_{2}=0.2568, \quad \mathrm{w}_{3}=0.2922, \quad \mathrm{w}_{4}=0.2188
$$

So the final reliability of the agricultural biological disaster prediction is

$$
\mathrm{R}_{\mathrm{s}}=\mathrm{w}_{1} \mu_{1}+\mathrm{w}_{2} \mu_{2}+\mathrm{w}_{3} \mu_{3}+\mathrm{w}_{4} \mu_{4}=0.7418
$$

\section{Conclusion}

Aiming at the practical problems of reliability evaluation on the agricultural biological disaster forecast, this paper introduced evidence theory and combined the expert experience. Evidence theory is to deal with the uncertainties caused by "not sure", "don't know" and uses the trust function as a metric. With the combination of the reliability evaluation and the exploration in fusion expert experience, this paper obtained the good effects. In the expert experience fusion, a lot of problems will be encountered, such as the express form of expert experience, the credibility of the expert experience, the correlation between expert experience and the experience conflict. To solve these problems well is the critical content in reliability assessment of information fusion.

\section{Acknowledgments.}

Project supported by the ResearchScience \& Technology Bureau of Hunan Province,China(Grant No. 2014FJ6046 ).

\section{References}

1. Booker J. M., Meyer M. A. Elicitation and analysis of expert judgment[J]. Encyclopedia of Statistical Sciences,(Samuel Kotz, editor), 2002.

2. Booker J. M., Meyer M. A.Uncertainty quantification: method and examples from probability and fuzzy theories[C]. World Automation Congress, 2002(13):135-140.

3. Dey D. K, Liu J. Prior elicitation from expert opinion: an interactive approach. University of Connecticut Division of Biostatistics[D]. Submitted and Ready for submission, 2004.

4. Szwed P. S., Drop J. R. A Bayesian model for rare event risk assessment using expert judgment about paired scenario comparisons[C]. ASEM National Conference Proceedings, 2002:444-453.

5. Hora S., Jensen M. Expert judgment elicitation[R]. SSIrapport:2002:19,ISSN 0282-4434,2002. 
International Conference on Engineering Management, Engineering Education and Information Technology (EMEEIT 2015)

6. Bram Wisse, Tim Bedford, John Quigley. Expert judgment combination using moment methods[J]. Reliability Engineering and System Safety, 2007:1-25. 